\title{
Ubiquitous expressed transcript promotes tumorigenesis by acting as a positive modulator of the polycomb repressive complex 2 in clear cell renal cell carcinoma
}

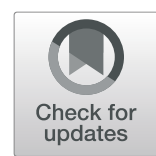

\author{
Jin Zeng ${ }^{1,2}$, Wei Xiang $^{3}$, Yucong Zhang ${ }^{1,4}$, Chunhua Huang ${ }^{3^{*}}$, Ke Chen ${ }^{1 *}$ (D) and Zhiqiang Chen ${ }^{1}$
}

\begin{abstract}
Background: The ubiquitous expressed transcript (UXT) plays a key role in various tumors by regulating transcriptional activity of multiple transcription factors, including androgen receptor (AR). However, the role of UXT in clear cell renal cell carcinoma (ccRCC) is still unknown.

Methods: Yeast two-hybrid screening, GST pull-down and co-immunoprecipitation assays were performed to detect the interacting protein of UXT. Chromatin immunoprecipitation (ChIP) was performed to investigate the levels of histone H3 lysine 27 trimethylation at the HOXA9 promoters. CCK-8 assays, colony formation assays and Transwell assays were performed to detect the proliferation, colony formation, migration and invasion of renal cancer cells. Quantitative PCR analysis was performed to detect the expressions of UXT in human ccRCC samples.

Results: The enhancer of zeste homolog $2(E Z H 2)$ is a novel UXT interacting protein and UXT interacts with EZH2 in the nucleus. In addition, UXT interacts with the polycomb repressive complex 2 (PRC2) through directly binding to EZH2 and suppressor of zeste 12 homolog (SUZ12), but not to embryonic ectoderm development (EED). Moreover, the UXT activates EZH2 histone methyltransferase activity by facilitating EZH2 binding with SUZ12. We further provided striking evidences that knockdown of UXT inhibits proliferation, colony formation, migration and invasion of renal cancer cells, in an EZH2-dependent manner. Importantly, the upregulation of UXT expression was observed in clinical ccRCC samples, and the high expression level of UXT was associated with advanced stage, distant metastasis and poor overall survival in patients with ccRCC.
\end{abstract}

Conclusion: The UXT is a novel regulator of the PRC2 and acts as a renal cancer oncogene that affects the progression and survival of ccRCC patients.

Keywords: UXT, EZH2, PRC2, HMTase activity, ccRCC, Cell migration, Cell invasion

\section{Background}

Renal cell carcinoma (RCC) is a common urologic tumor, with 338,000 new cases and accounts for about 144,000 deaths worldwide in 2012 [1]. The most prevalent RCC is clear cell renal cell carcinoma (ccRCC) which accounts for about $70-80 \%$ of RCC [2]. Previous

\footnotetext{
* Correspondence: hch_bms@hbtcm.edu.cn; kechen@hust.edu.cn

${ }^{3}$ College of Basic Medicine, Hubei University of Traditional Chinese Medicine, Wuhan 430065, People's Republic of China

${ }^{1}$ Department of Urology, Tongji Hospital, Tongji Medical College, Huazhong University of Science and Technology, Wuhan 430030, People's Republic of China

Full list of author information is available at the end of the article
}

studies have identified many driver genes that associated with ccRCC, including VHL [3], PBRM1 [4, 5], BAP1 [6], SETD2 [3, 7], TCEB1 [3, 8], KDM5C [3, 9], HIF1A [10], HIF2A [11, 12] and PTEN [3]. In spite of extensive ongoing researches, mechanisms underlie the progression of ccRCC are still not fully understood.

The ubiquitous expressed transcript (UXT), also named as androgen receptor trapped clone 27 (ART27), is expressed widely in all tissues of both human and mouse [13]. The best-known function of UXT is regulating multiple transcription factors, including AR, GATA4, NF-kB, and EVI1 [14-18]. It was shown that

(c) The Author(s). 2019 Open Access This article is distributed under the terms of the Creative Commons Attribution 4.0 International License (http://creativecommons.org/licenses/by/4.0/), which permits unrestricted use, distribution, and reproduction in any medium, provided you give appropriate credit to the original author(s) and the source, provide a link to the Creative Commons license, and indicate if changes were made. The Creative Commons Public Domain Dedication waiver (http://creativecommons.org/publicdomain/zero/1.0/) applies to the data made available in this article, unless otherwise stated. 

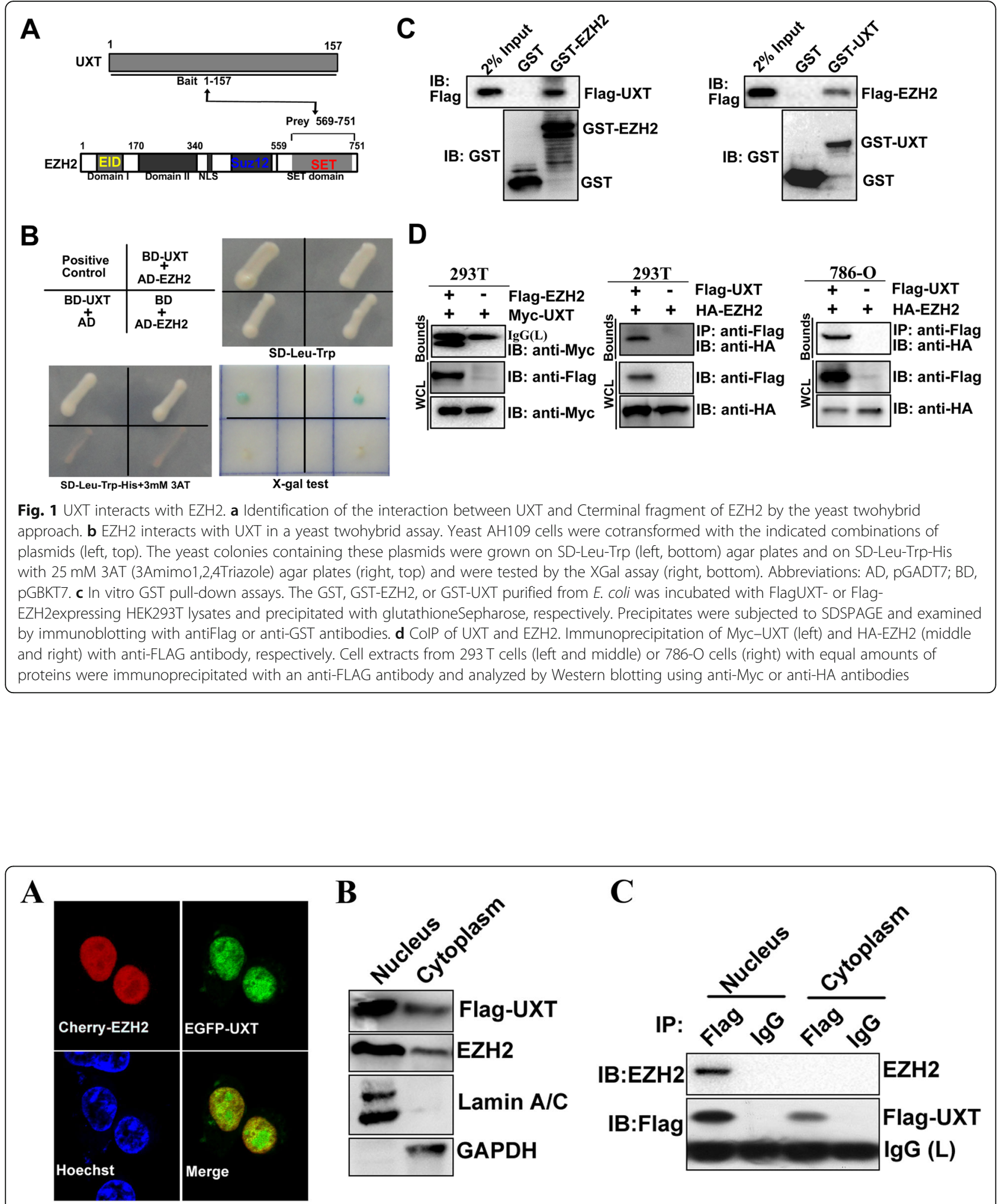

Fig. 2 UXT interacts with EZH2 in the nucleus. a Confocal microscopy shows the co-localization of EZH2 with UXT. 293 T cells were transfected with the EGFP-UXT and mCherry-EZH2 plasmids. The nuclei were stained with Hoechst 33258. b Western blot analysis confirmed the predominant expression of UXT and EZH2 protein in the nucleus of 293 T cells. c Endogenous EZH2 interacts with UXT in the nucleus. FLAG-UXT was transfected into $293 \mathrm{~T}$ cells. The nuclear and cytoplasmic fractions were isolated. Equal amounts of the fractions were immunoprecipitated with either control IgG or anti-FLAG antibody, followed by probing with an anti-EZH2 antibody 


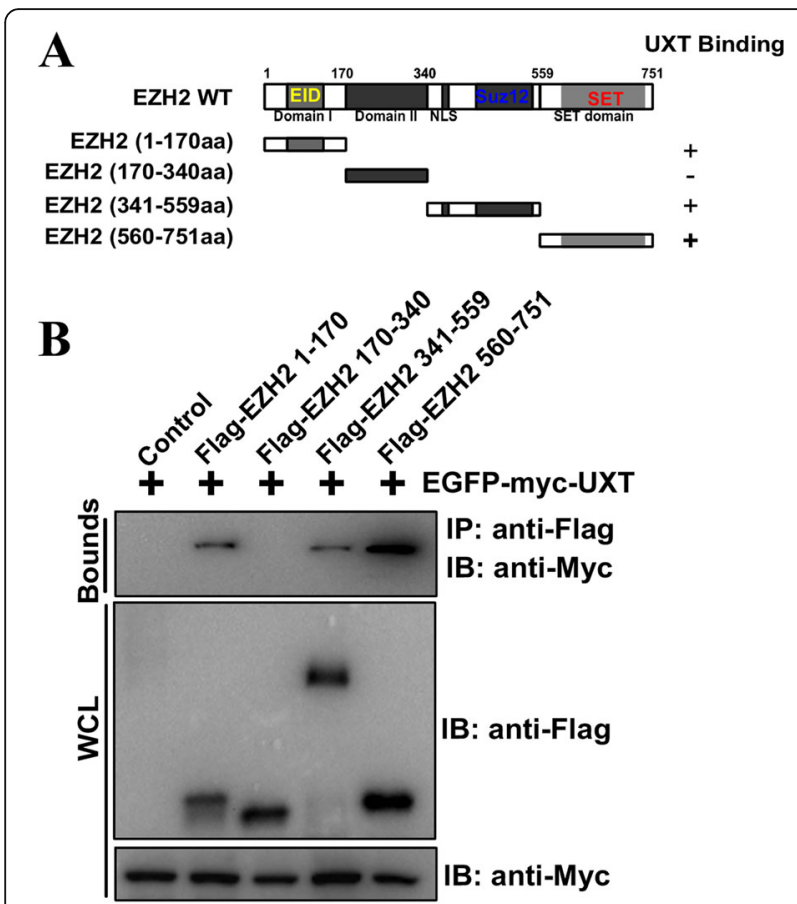

Fig. 3 Identification of the EZH2 domains required for interaction with UXT. a Schematic representation of various deletion mutants of Flag-EZH2 used for co-immunoprecipitation analyses. b EGFP-mycUXT was co-transfected with various Flag-EZH2 mutated plasmids and followed by Flag-immunoprecipitations and immunoblotting with Myc antibody

UXT can regulate expressions of AR downstream genes as a co-factor, by interacting with VHL and URI/RMP $[13,19]$. In addition, UXT regulates cell viability by interacting with centrosome [20]. Other investigations suggested UXT to be a component of the TNF receptor signaling complex, which plays a critical role in antiviral pathway [21, 22]. Deregulations of AR, GATA4, NF- $\mathrm{KB}$ and EVI1 signaling pathways are key factors that contribute to the progression of various malignancies. Although UXT was found ubiquitously expressed in human tissues, UXT expression is upregulated in multiple tumor tissues, including colorectal cancer [23], sarcoma [18], and breast tumor [24]. Thus, UXT has been thought to be a pro-oncogene in human cancer. However, McGilvray et al. report that UXT suppresses EVI1-mediated cell transformation [17]. It has also been reported that UXT is downregulated in prostate cancer tissue and its over-expression inhibited the prostate cancer cell growth [25-27]. Thus, UXT may both promote and suppress tumorigenesis, depending on the tumor types and microenvironments.

In the present study, the enhancer of zeste homolog 2 (EZH2) was identified as a novel UXT interacting protein by using yeast two-hybrid screening $(\mathrm{Y} 2 \mathrm{H})$. Besides, we demonstrated that UXT interacts with the polycomb repressive complex 2 (PRC2) through directly binding to EZH2 and suppressor of zeste 12 homolog (SUZ12), but not to embryonic ectoderm development (EED). UXT promotes the formation of PRC2 complex and increases EZH2 histone methyltransferase (HMTase) activity, which subsequently inhibits the expression of many tumor suppressor genes such as DAB2IP and HOXA9. Our data also showed that the expression of UXT was upregulated in ccRCC tissues compared with paracarcinoma tissues. Moreover, in RCC cells, the knockdown of UXT resulted in the inhibition of cell proliferation, colony formation, and cell migration. We also found that the effect of UXT depletion on these three activities was reversed by concomitant EZH2 overexpression. Strikingly, we also found that overexpression of UXT correlates with advanced TNM stage, distant metastasis, and poor survival in patients with ccRCC. Collectively, our investigation reveals that UXT is a new regulator of the $\mathrm{PRC} 2$ complex and is essential for its tumor promotion function in ccRCC.

\section{Methods \\ Reagents}

Flag, HA, and Myc, EZH2, Lamin A/C, DAB2IP, HOXA9, Histone H3, H3K27me3 (histone H3 lysine 27 trimethylation), and GAPDH antibodies were previously described [28-31]; EZH2 knockout and wild-type 786-O cells were previously described [30]. Glutathione Sepharose 4B resin (17-0756-01) was purchased from GE Healthcare.

\section{Clinical tissue sample collection}

Tumor tissues and normal adjacent tissues from pathologically and clinically confirmed ccRCC patients were kept in liquid nitrogen for total RNA extraction after radical nephrectomy. Written informed consent was obtained from all patients. This study was approved by the Tongji hospital of Tongji Medical College, Huazhong University of Science and Technology (Wuhan, China) ethics review committee.

\section{Constructs}

The $\mathrm{Y} 2 \mathrm{H}$ bait plasmid BD-UXT, mammalian expression plasmids for Flag- or HA- tagged EZH2, SUZ12, and EED were constructed as previously described $[13,30]$. Mammalian expression plasmids for human Cherry-, EGFP-, Flag-, GST-, or HA-tagged EZH2 and UXT and its truncated mutants were constructed by standard molecular biology techniques. PCDH-H1, which contains a lentiviral vector backbone, was constructed by subcloning the $\mathrm{H} 1$ promoter of pSilencer5.1-H1 Retro (Invitrogen) into the ClaI and NotI sites of pCDH-CD513B-1 (System Biosciences). Gene-specific shRNA target sequence was synthesized, annealed and cloned into the BamHI and NotI sites of the PCDH-H1 plasmid. 

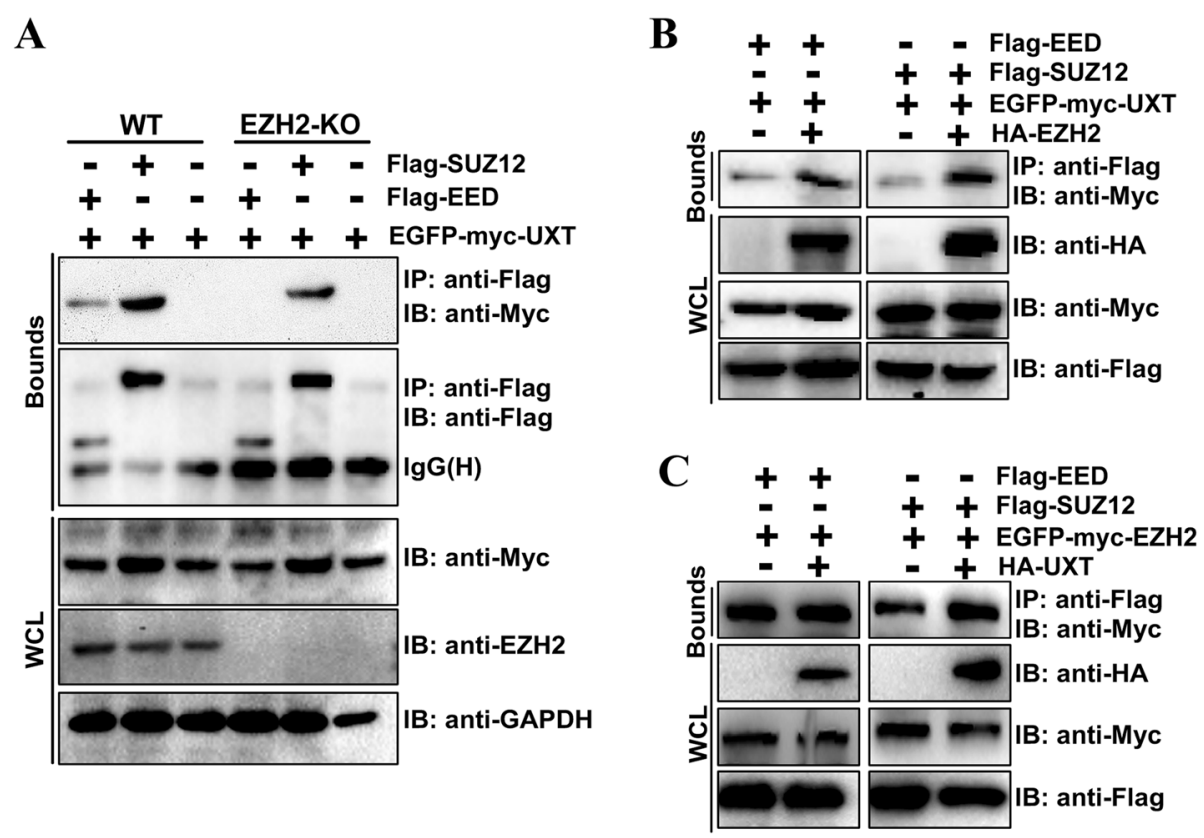

Fig. 4 UXT promotes EZH2-SUZ12 interaction. a UXT interacts with SUZ12, but not with EED, in EZH2-knockout cells. Cotransfection of EGFP-mycUXT into 786-O or EZH2-knockout 786-O cells was performed, together with Flag-SUZ12, Flag-EED, or an empty control plasmid psi-Flag, respectively. $48 \mathrm{~h}$ after transfection, the whole cell lysate was extracted for co-immunoprecipitation with anti-Flag, followed by probing with antiMyc. b EZH2 facilitates UXT binding with the other PRC2 components SUZ12 and EED. 293 T cells were co-transfected with the indicated combinations of plasmids. Cell lysates were precipitated with anti-Flag antibody and immunoblotted with anti-Myc antibody. c UXT binds to EZH2 and promotes the interaction between EZH2 and SUZ12, but has no effect on EZH2-EED interaction. 293 T cells were co-transfected with the indicated combinations of plasmids. Cell lysates were precipitated with anti-Flag antibody and immunoblotted with anti-Myc antibody

The primers for making these constructs were listed in Additional file 1: Table S1. All plasmids were verified by sequencing.

\section{RT-PCR, real-time PCR, and ChIP-qPCR analysis}

Total RNAs extraction and cDNAs synthesis were performed according to the instructions of manufacturer using Trizol (Invitrogen) and ReverTra Ace qPCR RT Kit (TOYOBO), respectively. SYBR Green qPCR Master Mix (Roche) using ABI ViiA7 Real-time PCR System (Applied Biosystems) were used to perform real-time PCR. ChIP (chromatin immunoprecipitation) assays were performed as previously described to investigate the levels of H3K27me3 at the HOXA9 promoters [30]. Final analysis was performed using qPCR and shown as fold enrichment of the HOXA9 gene promoter.

\section{$\mathrm{Y} 2 \mathrm{H}$ analysis}

The detail of $\mathrm{Y} 2 \mathrm{H}$ screening was described previously [13]. BD-UXT was transformed into yeast strain AH109 as the bait. Then, these yeast colonies were collected and transformed with cDNA library (Clontech). Positive clones' identification was performed by using SD-LeuHis-Ade selection plates and $\beta$-galactosidase assay. Positive plasmids were amplified in Escherichia coli DH5 $\alpha$ and isolated by Endo-Free Plasmid Mini Kit II
(Omega). BD-UXT or pGBKT7 control plasmid was cotransformed with these positive plasmids to confirm the specific interactions.

\section{GST pulldown and co-immunoprecipitation assays}

GST pull-down assays was performed as previously described [32]. Escherichia coli cells were transformed with GST, GST-UXT, or GST-EZH2 plasmid and then lysed in NETN buffer. The GST recombinant proteins' purification was performed using the glutathione-Sepharose $4 \mathrm{~B}$ resin. Next, these proteins were incubated with the lysates from HEK293T cells, which were transfected with FlagUXT or Flag-EZH2. Co-immunoprecipitation experiments were performed by using HEK293T cells or 786-O cells to analyze protein interactions [28].

Cell proliferation, colony formation, cell migration, and invasion assays

After seeding 1000 cells per well in 6-well plates, colony formation assays were measured twelve days later. According to the manufacturer's instructions, the CCK-8 (Dojindo Laboratories) was used to detect cell proliferation. Uncoated and Matrigel-coated Transwell inserts were used to perform cell migration and invasion assays. All experiments were performed in three independent experiments. 
A

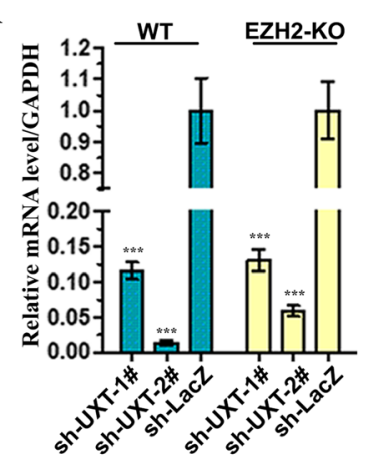

D

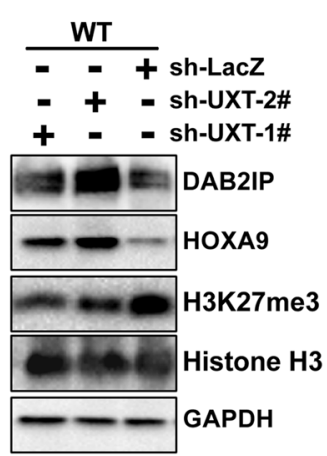

B

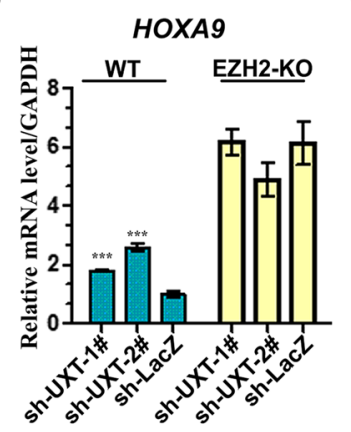

$\mathbf{E}$

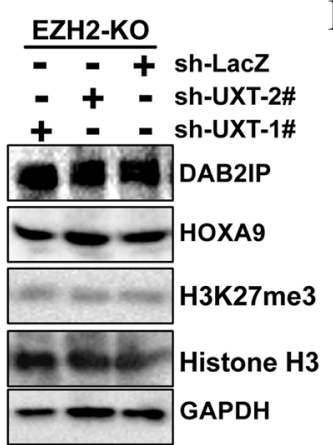

C

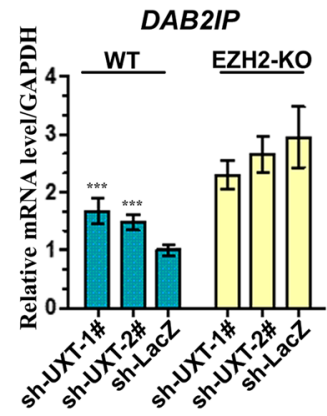

$\mathbf{F}$

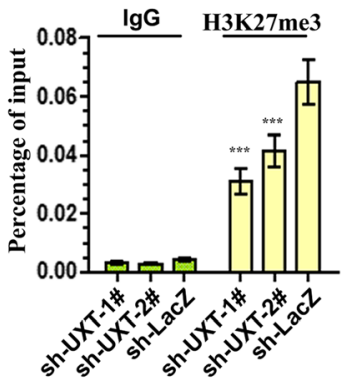

Fig. 5 UXT promotes EZH2 HMTase activity. a Real-time PCR analysis of UXT mRNA showed efficient UXT knockdown by shRNA expression in 786-O and EZH2-knockout 786-O cells. b, c Real-time quantitative PCR shows that UXT knockdown promotes the expression of endogenous EZH2 target genes (HOXA9 and DAB2IP) in EZH2 WT cells, but not in EZH2-knockout cells. Data are plotted as the mean \pm SD of three independent experiments. d, e Western blot analysis shows upregulation of DAB2IP and HOXA9 and downregulation of histone H3K27me3 in EZH2 WT 786-O cell lines (D), but not in EZH2-knockout cells(E). Total histone H3 and GAPDH were shown as control. $\mathbf{f}$ ChIP-qPCR analysis of H3K27me3 enrichment on HOXA9 gene promoter in UXT knockdown cells. UXT positive regulates H3K27me3 levels at PRC2 target loci in 786-O cells

\section{Analysis of TCGA ccRCC samples}

The TCGA data about mRNA (RNA Seq v2) expression levels in ccRCC patients were obtained from https://confluence.broadinstitute.org. Clinical data and recent follow-up data of ccRCC were downloaded from an integrated TCGA Pan-Cancer Clinical Data Resource [33]. In addition, samples lacking information about TNM, or grading and samples revealed the non-ccRCC phenotype were excluded from the analyses [34]. Differential expression between ccRCC and normal kidneys was calculated using the downloaded RSEM values. Survival curve was plotted using the Kaplan-Meier method and compared with the log-rank test.

\section{Statistical analysis}

The data is presented as the means $\pm \mathrm{SD}$. Comparisons between two groups were performed using an unpaired Student's t-test. Three levels of significance were used $\left(" p<0.05, * * p<0.01\right.$, and $\left.{ }^{* * * *} p<0.001\right)$.

\section{Results}

Identification of EZH2 as a novel UXT binding protein

UXT is associated with human tumorigenesis [20, 23]. However, the regulatory mechanisms associated with UXT- mediated tumorigenesis are still not well understood. To further investigate the downstream protein of UXT, a yeast two-hybrid screen was performed with UXT as bait protein. A total of 14 positive clones from $2 \times 10^{6}$ transformants were identified (Additional file 2: Table S2). One of these clones encodes partial C-terminal region of EZH2 (Fig. 1a). To further verify the interaction between EZH2 and UXT, BDUXT and ADEZH2 were co-transformed into AH109 yeast cells (Fig. 1b, left, top). Reporter gene was found to be significantly activated after co-expression of UXT and EZH2 (Fig. 1b, bottom). To determine the direct interaction between UXT and EZH2, a GST pull-down assay was used. As expected, UXT protein was detected after incubated with GST-EZH2 but not GST, which indicated the specific binding between UXT and EZH2 (Fig. 1c, left). In a reciprocal assay, EZH2 was also detected to bind with GST-UXT (Fig. 1c, right). Further co-immunoprecipitation assays showed that Myc-UXT was co-precipitated efficiently with Flag-EZH2 and HA-EZH2 was also co-precipitated efficiently with Flag-UXT in $293 \mathrm{~T}$ cells (Fig. 1a).

\section{UXT associated with EZH2 in the nucleus}

For further investigation of intracellular colocalization and interaction of UXT and EZH2, EGFP-tagged UXT and 


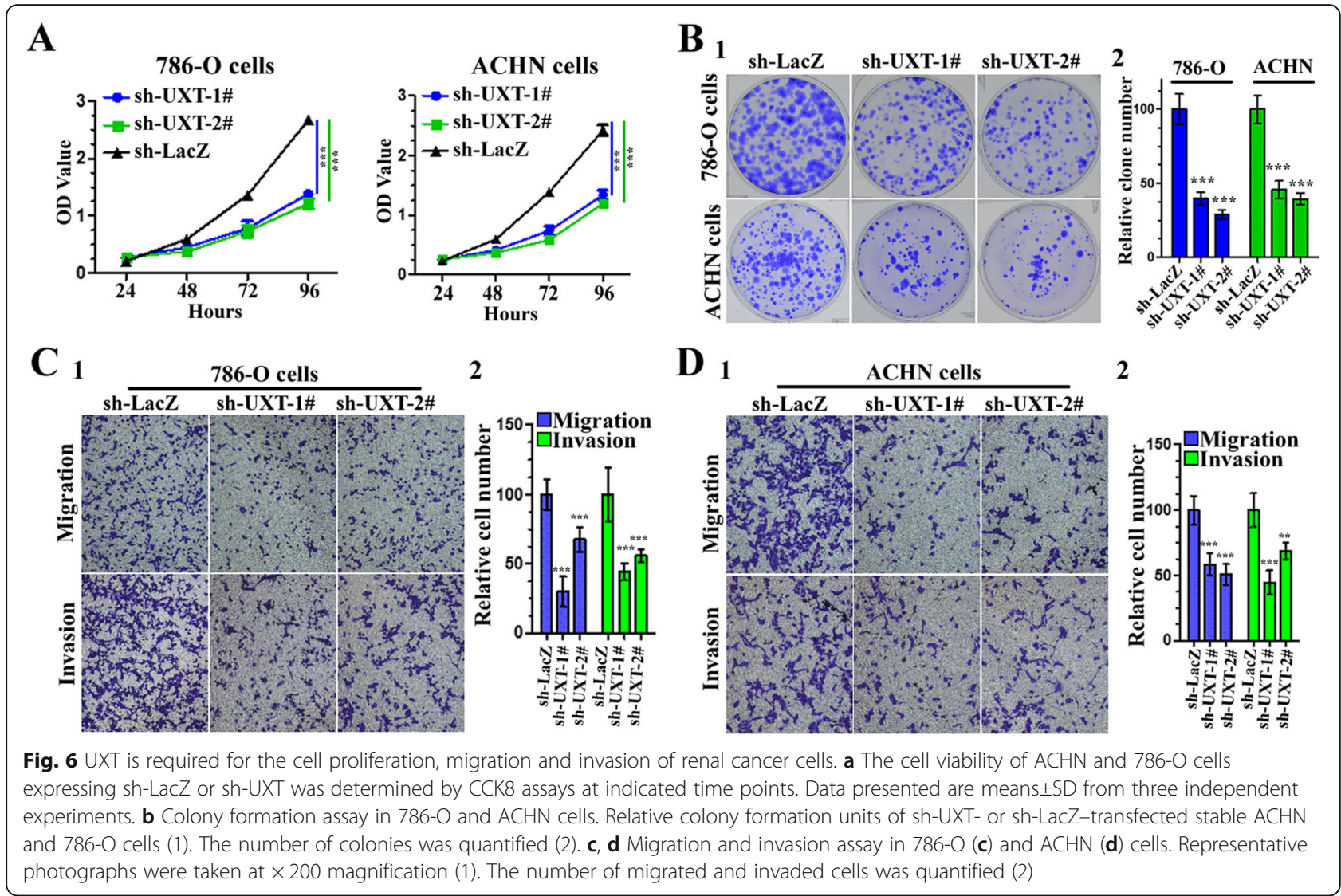

mCherry-tagged EZH2 were co-expressed in $293 \mathrm{~T}$ cells by transfecting recombinant plasmids. Co-localization experiments showed that UXT and EZH2 were colocalized in the nucleus (Fig. 2a). Further cell fractionation and Western blot analysis showed that both Flag-UXT and endogenous EZH2 are predominantly expressed in the nucleus (Fig. 2b). In addition, immunoprecipitation assays showed that endogenous EZH2 was co-precipitated efficiently with FlagUXT in nucleic nucleus but not in cytoplasmic components (Fig. 2c). Taken together, these results confirm that UXT interacts with $\mathrm{EZH} 2$ in the nucleus.

\section{Identification of the interacting domains of EZH2 with UXT}

Next, we delineated the domains that respond for the interaction between UXT and $\mathrm{EZH} 2$ by co-expression of EGFP-myc-UXT and Flag-EZH2 deletion mutants. Considering UXT is a small protein and only contains one $\alpha$ domain [35], only UXT interacting domains of $\mathrm{EZH} 2$ were investigated. A series of EZH2 deletion mutants, which contain the Domain I [amino acids (aa) 1-170], Domain II [aa 170-340], SUZ12 binding domain [aa 341-559], and SET domain [aa 560-751], was co-expressed with EGFP-myc-UXT in HEK293T cells for co-immunoprecipitation assays (Fig. 3a). These results indicate that Domain II of EZH2 is insufficient to interact with UXT, but Domain I and SUZ12-binding domains showed decreased binding to UXT (Fig. 3b).

\section{UXT facilitates EZH2 binding with SUZ12}

EZH2 is classically associated with PRC2 complex, and both PRC2 components, including SUZ12 and EED. These three PRC2 components are required for its HMTase activity. Thus, we examined whether UXT interacts with SUZ12 and EED. Co-immunoprecipitation assays of Flag-EED or Flag-SUZ12 with EGFP-myc-UXT in 786-O cells showed that UXT was coprecipitated efficiently with both EED and SUZ12 (Fig. 4a; lanes 1-3), which suggested that UXT is associated with the PRC2 complex. However, knockout of EZH2 led to disruption of EED binding to UXT but did not disrupt the binding to SUZ12 (Fig. 4a; lanes 4 and 5). These results suggested that UXT are directly associated with EZH2 and SUZ12, and EZH2 mediates the interaction between UXT and EED. Furthermore, there was weaker binding between SUZ12 and UXT in EZH2 knockout cells (Fig. 4a; lanes 5 versus 2), comparing to wild-type cells, which suggested that EZH2 facilitates UXT binding to SUZ12. Indeed, the overexpression of EZH2 resulted in the promotion of EGFP-myc-UXT binding to EED or SUZ12 in $293 \mathrm{~T}$ cells (Fig. 4b). Further investigation 

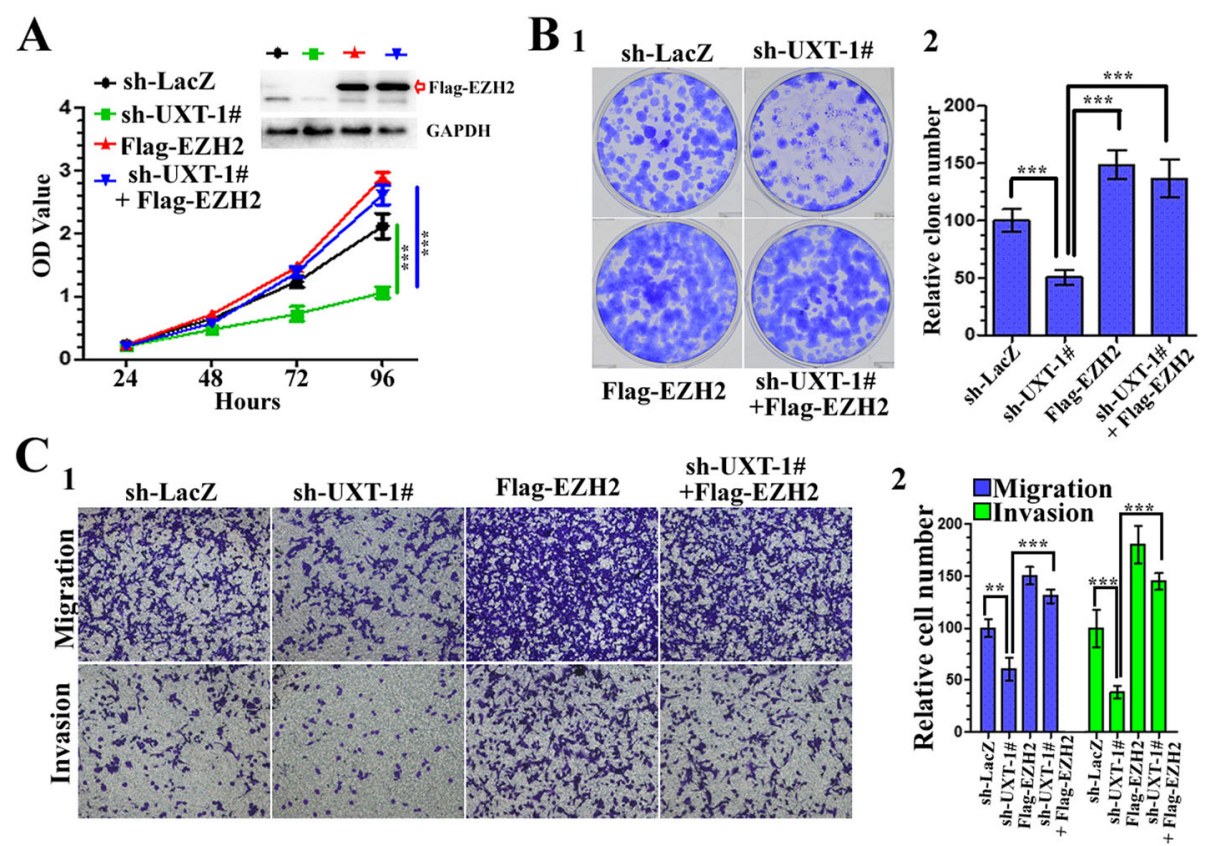

Fig. 7 UXT regulates human renal cancer cell proliferation, migration and invasion via EZH2. a 786-O cells were infected with different combinations of lentivirus as indicated. $72 \mathrm{~h}$ after infection, CCK8 assay was utilized to quantify cell viability at each time point. Data are plotted as the mean \pm SD of 3 independent experiments. Western blot analysis of the cells was performed to evaluate the expression of EZH2. GAPDH is used as an internal control. b After infection as (a), after infection, colony formation assay was performed to estimate the anchorage-independent growth ability of 786-O cells. Images were taken at 2 weeks after 2 weeks (1). The number of colonies was quantified (2). c After infection as (a), cells were used for Transwell assays. Representative photographs were taken at $\times 200$ magnification (1). Number of migrated and invaded cells was quantified in 4 random images from each treatment group. Results are the mean \pm SD from 3 independent experiments plotted as percent (\%) migrating and invading cells relative to sh-LacZ treatment

showed that overexpression of UXT had no effect on interaction between EZH2 and EED (Fig. 4c; lanes 1 and 2). However, ectopically expressed UXT was found to strengthen EZH2-SUZ12 interaction (Fig. 4c; lanes 3 versus 4). Taken together, these data support the hypothesis that UXT may promote EZH2 HMTase activity by contributing the binding between EZH2 and SUZ12.

\section{UXT promotes EZH2 HMTase activity}

Given that EZH2 is histone H3 lysine 27 (H3K27) methyltransferase, which is associated with transcriptional repression, we sought to investigate the role of UXT in EZH2's transcriptional repressor function. We stably inhibited UXT in both EZH2-WT and EZH2-KO 786-O cells. shUXT-1\# and sh-UXT-2\# incorporated cells were found to significantly reduced expression levels of UXT, compared with control cells (Fig. 5a). Next, we evaluated the expressions of EZH2-targets, including HOXA9 and DAB2IP, at both mRNA and protein levels by RT-qPCR and Western Blot. We found that HOXA9 and DAB2IP were upregulated by shRNA-mediated UXT knockdown in the EZH2WT cells (Fig. 5b-c) but not in the EZH2-KO cells (Fig. 5bc), which suggested that UXT depletion can promote the transcription of HOXA9 and DAB2IP through EZH2. Consistent with these results, knockdown of UXT in the
EZH2-WT cells inhibited the EZH2 histone methyltransferase activity, indicated by the decrease of H3K27 methylation and increase of protein levels of HOXA9 and DAB2IP (Fig. 5d). In contrast, UXT depletion didn't result in increase of expression levels of HOXA9 and DAB2IP in the EZH2-KO cells (Fig. 5e). In addition, by using quantitative chromatin immunoprecipitation (qChIP) assays, lower levels of H3K27 trimethylation(H3K27me3)on the HOXA9 promoter was detected in UXT knockdown cells comparing to the control cells (Fig. 5f). Taken together, these results suggest that UXT promotes formation of PRC2 and results in promotion of its HMTase activity.

\section{UXT is a renal cancer oncogene}

Since both UXT and EZH2 have been implicated in tumorigenesis, we next investigated whether UXT might regulate renal cancer tumorigenesis through modulating EZH2 HMTase activity. We and other groups have demonstrated that EZH2 function as an oncogene in ccRCC $[30,36-38]$. However, the role of UXT in ccRCC is still under exploration. We first examined the tumorigenicity of UXT in renal cancer cells. As expected, UXT depletion inhibited cell proliferation and anchorage-dependent growth in $\mathrm{ACHN}$ 


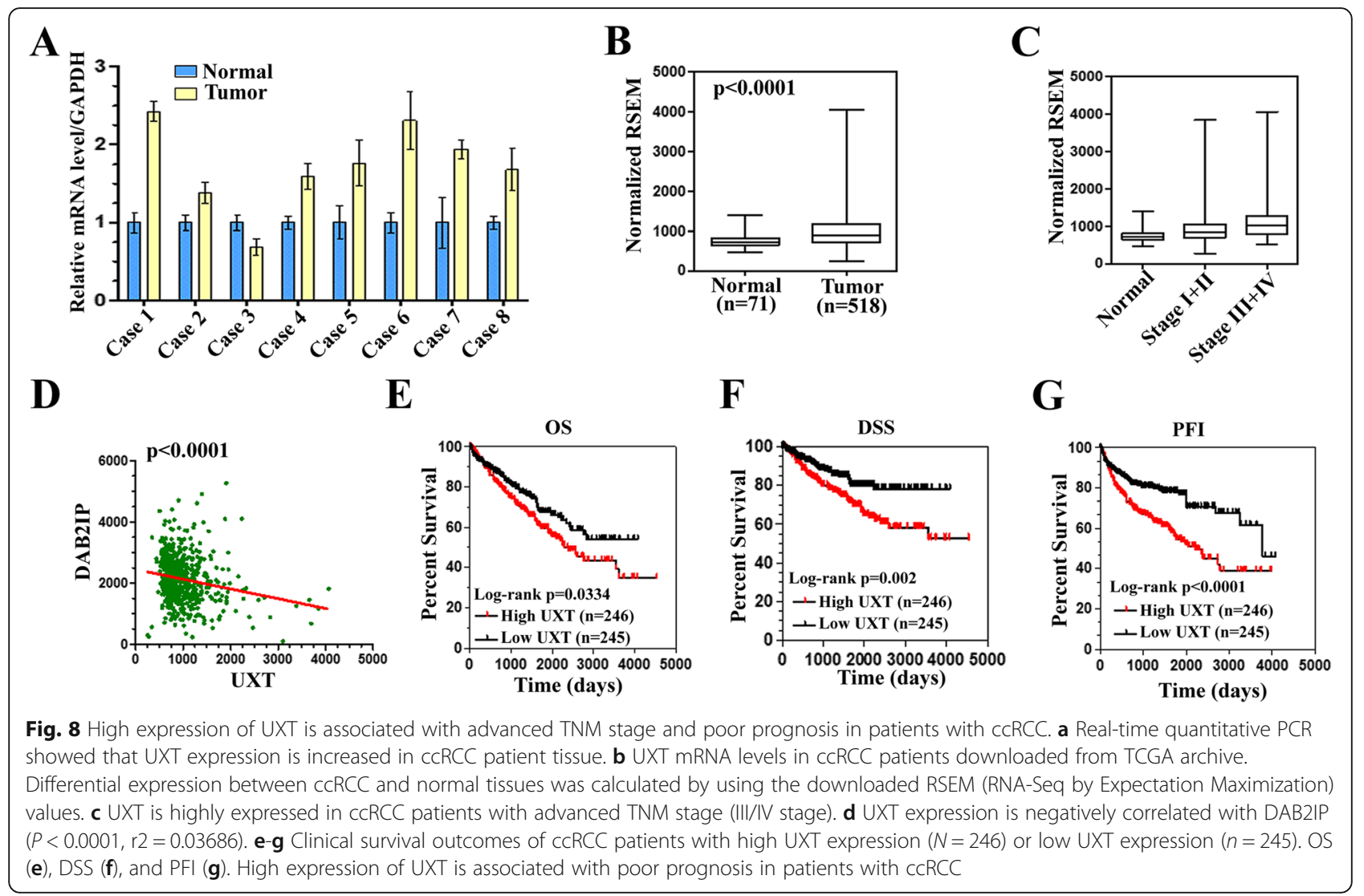

and 786-O cells, as judged by CCK 8 assays and colony formation (Fig. 6a-b). Secondly, knockdown of UXT inhibited migration and invasion of $\mathrm{ACHN}$ and 786-O cells (Fig. 6c-d). Together, these findings indicate that UXT is a potential candidate protooncogene in ccRCC.

Next, we tested whether exogenous EZH2 could overturn the suppressive effects of UXT knockdown on the tumorigenicity. We found that effects of UXT depletion on proliferation, migration, and invasion were reversed by concomitant EZH2 overexpression, which suggested that the effects of UXT on cell migration and invasion are mediated, at least in part, through EZH2 (Fig. 7). This finding supports the hypothesis that UXT promotes tumor progression (at least partially) through EZH2.

\section{UXT is increased in ccRCC patients and correlates negatively with survival}

To further investigate the role of UXT as a pro-oncogene, we examined its expression and clinical relevance in patients with ccRCC. First, qPCR analysis was performed to detect the mRNA level of UXT in 8 paired renal cancer samples and adjacent normal tissues. It was found that 7 of 8 tumors collected from renal cancer specimens have a significantly increased UXT level compared with corresponding normal controls (Fig. 8a). We next analyzed the expression levels of UXT in 518 tumors and 71 adjacent normal tissues of ccRCC from The Cancer Genome Atlas (TCGA) archive, and found that tumors have a significantly increased UXT level compared with adjacent normal tissues (Fig. 8b). In addition, the UXT expression was significantly positive correlated with TNM stage $(P=0.0003)$ (Table 1$)$. UXT was increased 1.26-fold $(\mathrm{P}=0.0003)$ in low-stage (Stages I and II) and 1.48-fold $(P<0.0001)$ in advanced-stage (Stages III and IV) tumors, demonstrating that UXT expression is associated with advanced tumor stages (Fig. 8c). Interestingly, UXT expression negatively correlated with DAB2IP $\left(\mathrm{P}<0.0001, \mathrm{r}^{2}=0.03686\right.$, Spearman correlation) (Fig. 8d), corroborating our observation that UXT promotes the EZH2 HMTase activity, which inhibits the transcription of DAB2IP. Importantly, increased UXT expression is probably associated with advanced distant metastasis and poor survival in patients with ccRCC (Table 1). A higher expression of UXT was closely associated with poor overall survival (OS) (Fig. 8e), disease-specific survival (DSS) (Fig. 8f), and progression-free interval (PFI) (Fig. 8g) in patients with ccRCC. These results indicate that UXT 
Table 1 Clinical characteristics and outcome of 491 ccRCC patients downloaded from TCGA according to UXT gene expression status

\begin{tabular}{|c|c|c|c|c|}
\hline \multirow[t]{2}{*}{ Characteristic } & \multirow{2}{*}{$\begin{array}{l}\text { Total }(n= \\
\text { 491) }\end{array}$} & \multicolumn{2}{|l|}{ UXT } & \multirow[t]{2}{*}{$P$} \\
\hline & & High $(n=246)$ & Low $(n=245)$ & \\
\hline Gender & & & & $P=0.0006$ \\
\hline Male & 313 & 175 & 138 & \\
\hline Female & 178 & 71 & 107 & \\
\hline Age & & & & $P=0.0643$ \\
\hline$\leq 60$ & 242 & 111 & 131 & \\
\hline$>60$ & 249 & 135 & 114 & \\
\hline Mean (SD) & $60.8(12.1)$ & $61.7(12.1)$ & $60.0(12.0)$ & \\
\hline Grade & & & & $P=0.0007$ \\
\hline G1-G2 & 223 & 92 & 128 & \\
\hline G3-G4 & 267 & 153 & 114 & \\
\hline GX & 3 & 1 & 3 & \\
\hline TNM Stage & & & & $P=0.0003$ \\
\hline$|-| \mid$ & 293 & 127 & 166 & \\
\hline III-IV & 198 & 119 & 79 & \\
\hline T Stage & & & & $P=0.0023$ \\
\hline $\mathrm{T} 1-\mathrm{T} 2$ & 210 & 139 & 171 & \\
\hline T3-T4 & 181 & 107 & 74 & \\
\hline Lymph node metastasis & & & & $P=0.6509$ \\
\hline No & 220 & 108 & 112 & \\
\hline N1 & 14 & 6 & 8 & \\
\hline NX & 258 & 132 & 126 & \\
\hline Distant metastasis & & & & $P=0.0431$ \\
\hline MO & 385 & 187 & 198 & \\
\hline M1 & 75 & 46 & 29 & \\
\hline$M X$ & 31 & 13 & 18 & \\
\hline Vital status & & & & $P=0.0009$ \\
\hline Dead & 163 & 99 & 64 & \\
\hline Alive & 328 & 147 & 181 & \\
\hline
\end{tabular}

Two-tailed chi-square test was done with the SPSS software program to determine the statistical significance of the level of expression of UXT in different Clinical characteristics, $P<0.05$ was considered significant

might be recognized as a prognostic factor for cancer survival. Taken together, these results validate the mechanistic link between increased UXT expression and ccRCC progression, which supported the conclusion that UXT is a renal cancer oncogene which affects the progression and survival of ccRCC patients.

\section{Discussion}

In the present study, we present a newly identified mechanism for UXT in ccRCC, where UXT regulates tumor progression by regulating PRC2 activity in renal cancer cells. By using yeast two-hybrid to identify protein partners of UXT, we uncovered interactions between UXT and EZH2 in PRC2 complex. Specifically, we suggest that UXT promotes EZH2 HMTase activity by promoting the formation of PRC2 complex. In a previous study [35], it was proposed that the UXT interacts with the EZH1-SUZ12 complex to regulate the transcription of NF- $\mathrm{kB}$ target genes, and demonstrated that UXT neither binds EZH2 nor regulates its HMTase activity in HCT116 cells. In this regard, we recognized that these results contradict ours and examined this issue from several perspectives. We demonstrated that UXT can bind to EZH2 by various assays, including yeast two-hybrid, GST pull down, and co-immunoprecipitation. Since protein-protein interactions (PPIs) are dynamic process, and sometimes, are specific to the cell type and cell cycle phase [39, 40], it is possible that UXTEZH2 interaction is context-dependent or/and cell-type 
specific. Further studies are necessary to elucidate the reason for this discrepancy. In addition, we found that multiple domain of EZH2 can interact with UXT. Since UXT contains one $\alpha$ domain and shows homology to prefoldin which functions as a co-chaperone and plays important role in protein folding [35], which suggests that UXT may regulate the protein folding of PRC2 complex.

UXT plays a significant role in regulating the progression of various tumors, including prostate and breast cancer [14, $15,17,24-27]$ and is classically known as both coactivator and corepressor protein that participates in the regulation of several transcription factors by direct interaction. UXT can interact with the $\mathrm{N}$ terminus of AR and facilitate receptor dependent transcriptional activation, which contributes to its role in AR-dependent prostate tumorigenesis [14, 25]. It has also been reported that UXT functions primarily as an AR corepressor and represses transcription of AR downstream genes [26]. UXT also modulates estrogen receptor$\alpha$ activity by interacting with LOX-PP in breast cancer cells [24]. In addition, UXT also interacts with many other wellknown tumor-associated transcription factors, such as NFкB, EVI1, GATA4, FOG2, NKX2.5, and Foxp3 [15-17, 41], which suggests that UXT may regulate tumorigenesis though multiple signaling pathways. The mechanism by which UXT affects tumorigenesis remains to be defined.

PRC2 complex catalyzes the mono-, di-, and trimethylation of lysine 27 of histone H3, which plays an important role in the regulation of multiple biological processes, including tumor initiation and progression [42, 43]. EZH2 is the catalytic subunit of PRC2, which has been shown to be dysregulated in various different cancer types, including ccRCC [30, 36-38]. Mechanistically, EZH2 methylates $\mathrm{H} 3 \mathrm{~K} 27$ to promote transcriptional silencing of many tumor suppressor genes [44]. In addition to histone, many other non-histone proteins also methylated by EZH2 [45-49], and the interaction between EZH2 and these non-histone substrates plays important role in the development and progression of a variety of cancers. Interestingly, EZH2 serves as a coactivator of AR through direct interaction to facilitate its oncogenic function in cells of castration-resistant prostate cancer [49]. Furthermore, EZH2 also functions either as a novel positive or negative regulator of NF-kB depending on the ER status [50]. Given that UXT also functions as a transcriptional co-regulator of AR [14, 26], NF-kB [15, 18], and ER [24], it will be very interesting to determine whether UXT and EZH2 synergistically regulate the activity of these transcription factors.

In this study, knockdown of UXT demonstrated that this protein plays an essential role in ccRCC. More importantly, we found that EZH2 was sufficient to rescue the growth defects following UXT inhibition. Thus, our results support a model that UXT regulates the formation of PRC2 complex and plays an important role in repression of PRC2 target genes, therefore, promotes
ccRCC progression. Consistently, UXT is upregulated in renal clear cell carcinomas and highly negative correlates with DAB2IP expression. Furthermore, we analyzed the data of over 500 ccRCC patients retrieved from TCGA archive, and found that the expression level of UXT is clearly correlated with the grade, stage and metastasis of tumors. Interestingly, we also found that UXT was highly expressed among male ccRCC patients. As a coregulator of AR, UXT may also be up-regulated by AR in a feedback manner, which helps to explain the reason for higher expression level of UXT in male over female patient with ccRCC. Further elucidation of the mechanism of UXT in the promotion of EZH2 activity may provide new therapeutic targets for ccRCC.

\section{Conclusion}

In summary, we discovered a novel UXT/PRC2 complex that represses the expression of EZH2 target genes. And the UXT acts as a renal cancer oncogene that affects the progression and survival of ccRCC patients.

\section{Additional files}

Additional file 1: Table S1 The primers used in the study. (DOC $44 \mathrm{~kb}$ ) Additional file 2: Table S2 The potential UXT-interacting proteins identified by a yeast two-hybrid assay. (DOCX $19 \mathrm{~kb}$ )

\section{Abbreviations}

cCRCC: Clear cell renal cell carcinoma; EED: Embryonic ectoderm development; EZH2: Enhancer of zeste homolog 2; H3K27: Histone H3 lysine 27; H3K27me3: Histone H3 lysine 27 trimethylation; HMTase: Histone methyltransferase; PRC2: Polycomb repressive complex 2; RCC: Renal cell carcinoma; SUZ12: Suppressor of zeste 12 homolog; UXT: Ubiquitous expressed transcript;

\section{Acknowledgements}

We would like to thank all the patients for their contribution in this study.

\section{Authors' contributions}

$\mathrm{KC}$ and $\mathrm{CH}$ designed the study. JZ, $\mathrm{CH}$ and $\mathrm{KC}$ wrote the manuscript. JZ, WX and $Y Z$ performed experiments. JZ and $K C$ and $Z C$ analyzed data. JZ and $Y Z$ prepared the clinical samples. All authors read and approved the final manuscript.

\section{Funding}

This work was supported by National Natural Science Foundation of China [grant number 81772721, 81402105), and the Tongji Hospital Science Fund for Outstanding Young Scholars (2016YQ07). The funders had no role in study design, data collection and analysis, decision to publish, or preparation of the manuscript.

\section{Availability of data and materials}

The datasets used and analyzed during the current study are available from the corresponding author on reasonable request.

Ethics approval and consent to participate

This study was approved by the Tongji hospital of Tongji Medical College, Huazhong University of Science and Technology (Wuhan, China) ethics review committee. All human tumor tissues were obtained with written informed consent from patients prior to participation in the study. 


\section{Consent for publication}

Not applicable.

\section{Competing interests}

The authors declare that they have no competing interests.

\section{Author details}

'Department of Urology, Tongji Hospital, Tongji Medical College, Huazhong University of Science and Technology, Wuhan 430030, People's Republic of China. ${ }^{2}$ Department of Urology, the First Affiliated Hospital of Nanchang University, Nanchang 330000, People's Republic of China. ${ }^{3}$ College of Basic Medicine, Hubei University of Traditional Chinese Medicine, Wuhan 430065, People's Republic of China. ${ }^{4}$ Department of Geriatric, Tongji Hospital, Tongj Medical College, Huazhong University of Science and Technology, Wuhan 430030, People's Republic of China.

Received: 24 March 2019 Accepted: 20 August 2019

\section{Published online: 03 September 2019}

\section{References}

1. Rodrigues P, Patel SA, Harewood L, Olan I, Vojtasova E, Syafruddin SE, Zaini MN, Richardson EK, Burge J, Warren AY, et al. NF-kappaB-dependent lymphoid enhancer co-option promotes renal carcinoma metastasis. Cancer Discov. 2018;8(7):850-65.

2. Sato $Y$, Yoshizato $T$, Shiraishi $Y$, Maekawa S, Okuno Y, Kamura T, Shimamura T, Sato-Otsubo A, Nagae G, Suzuki H, et al. Integrated molecular analysis of clear-cell renal cell carcinoma. Nat Genet. 2013;45(8):860-7.

3. Creighton CJ, Morgan M, Gunaratne PH, Wheeler DA, Gibbs RA, Gordon Robertson A, Chu A, Beroukhim R, Cibulskis K, Signoretti S, et al. Comprehensive molecular characterization of clear cell renal cell carcinoma. Nature. 2013:499(7456):43-9.

4. Varela I, Tarpey P, Raine K, Huang D, Ong CK, Stephens P, Davies H, Jones D, Lin $M-L$, Teague J, et al. Exome sequencing identifies frequent mutation of the SWI/SNF complex gene PBRM1 in renal carcinoma. Nature. 2011; 469(7331):539-42.

5. Brugarolas J. PBRM1 and BAP1 as novel targets for renal cell carcinoma. Cancer J (Sudbury, Mass). 2013;19(4):324-32.

6. Pena-Llopis S, Vega-Rubin-de-Celis S, Liao A, Leng N, Pavia-Jimenez A, Wang S, Yamasaki T, Zhrebker L, Sivanand S, Spence P, et al. BAP1 loss defines a new class of renal cell carcinoma. Nat Genet. 2012;44(7):751-9.

7. Kanu N, Grönroos E, Martinez P, Burrell RA, Yi Goh X, Bartkova J, MayaMendoza A, Mistrík M, Rowan AJ, Patel H, et al. SETD2 loss-of-function promotes renal cancer branched evolution through replication stress and impaired DNA repair. Oncogene. 2015;34:5699

8. Hakimi AA, Tickoo SK, Jacobsen A, Sarungbam J, Sfakianos JP, Sato Y, Morikawa T, Kume H, Fukayama M, Homma Y, et al. TCEB1-mutated renal cell carcinoma: a distinct genomic and morphological subtype. Mod Pathol. 2015;28(6):845-53.

9. Rondinelli B, Rosano D, Antonini E, Frenquelli M, Montanini L, Huang D, Segalla S, Yoshihara K, Amin SB, Lazarevic D, et al. Histone demethylase JARID1C inactivation triggers genomic instability in sporadic renal cancer. J Clin Invest. 2015;125(12):4625-37.

10. Shen C, Beroukhim R, Schumacher SE, Zhou J, Chang M, Signoretti S, Kaelin WG Jr. Genetic and functional studies implicate HIF1alpha as a 14q kidney cancer suppressor gene. Cancer Discov. 2011;1(3):222-35.

11. Chen W, Hill H, Christie A, Kim MS, Holloman E, Pavia-Jimenez A, Homayoun F, Ma Y, Patel N, Yell P, et al. Targeting renal cell carcinoma with a HIF-2 antagonist. Nature. 2016;539(7627):112-7.

12. Cho H, Du X, Rizzi JP, Liberzon E, Chakraborty AA, Gao W, Carvo I, Signoretti S, Bruick RK, Josey JA, et al. On-target efficacy of a HIF-2alpha antagonist in preclinical kidney cancer models. Nature. 2016;539(7627):107-11.

13. Chen S, Chen K, Zhang Q, Cheng H, Zhou R. Regulation of the transcriptional activation of the androgen receptor by the UXT-binding protein VHL. Biochem J. 2013;456(1):55-66.

14. Markus SM, Taneja SS, Logan SK, Li W, Ha S, Hittelman AB, Rogatsky I, Garabedian MJ. Identification and characterization of ART-27, a novel coactivator for the androgen receptor $\mathrm{N}$ terminus. Mol Biol Cell. 2002;13(2):670-82.

15. Sun S, Tang Y, Lou X, Zhu L, Yang K, Zhang B, Shi H, Wang C. UXT is a novel and essential cofactor in the NF-kappaB transcriptional enhanceosome. J Cell Biol. 2007;178(2):231-44.
16. Carter DR, Buckle AD, Tanaka K, Perdomo J, Chong BH. Art27 interacts with GATA4, FOG2 and NKX2.5 and is a novel co-repressor of cardiac genes. PLoS One. 2014;9(4):e95253.

17. McGilvray R, Walker M, Bartholomew C. UXT interacts with the transcriptional repressor protein EVI1 and suppresses cell transformation. FEBS J. 2007:274(15):3960-71.

18. Qi M, Ganapathy S, Zeng W, Zhang J, Little JB, Yuan ZM. UXT, a novel MDMX-binding protein, promotes glycolysis by mitigating p53-mediated restriction of NF-kappaB activity. Oncotarget. 2015;6(19):17584-93.

19. Mita P, Savas JN, Djouder N, Yates JR 3rd, Ha S, Ruoff R, Schafler ED, Nwachukwu JC, Tanese N, Cowan NJ, et al. Regulation of androgen receptor-mediated transcription by RPB5 binding protein URI/RMP. Mol Cell Biol. 2011;31(17):3639-52.

20. Zhao H, Wang Q, Zhang H, Liu Q, Du X, Richter M, Greene MI. UXT is a novel centrosomal protein essential for cell viability. Mol Biol Cell. 2005; 16(12):5857-65.

21. Huang Y, Chen L, Zhou Y, Liu H, Yang J, Liu Z, Wang C. UXT-V1 protects cells against TNF-induced apoptosis through modulating complex II formation. Mol Biol Cell. 2011:22(8):1389-97.

22. Huang Y, Liu H, Ge R, Zhou Y, Lou X, Wang C. UXT-V1 facilitates the formation of MAVS antiviral signalosome on mitochondria. J Immunol. 2012; 188(1):358-66.

23. Yin J, Luo W, Zeng X, Zeng L, Li Z, Deng X, Tan X, Hu W. UXT-AS1-induced alternative splicing of UXT is associated with tumor progression in colorectal cancer. Am J Cancer Res. 2017:7(3):462-72.

24. Sanchez-Morgan N, Kirsch KH, Trackman PC, Sonenshein GE. UXT is a LOXPP interacting protein that modulates estrogen receptor alpha activity in breast Cancer cells. J Cell Biochem. 2017;118(8):2347-56.

25. Taneja SS, Ha S, Swenson NK, Torra IP, Rome S, Walden PD, Huang HY, Shapiro E, Garabedian MJ, Logan SK. ART-27, an androgen receptor coactivator regulated in prostate development and Cancer. J Biol Chem. 2004;279(14):13944-52

26. Nwachukwu JC, Mita P, Ruoff R, Ha S, Wang Q, Huang SJ, Taneja SS, Brown M, Gerald WL, Garabedian MJ, et al. Genome-wide impact of androgen receptor trapped clone-27 loss on androgen-regulated transcription in prostate cancer cells. Cancer Res. 2009;69(7):3140-7.

27. Nwachukwu JC, Li W, Pineda-Torra I, Huang HY, Ruoff R, Shapiro E, Taneja SS, Logan SK, Garabedian MJ. Transcriptional regulation of the androgen receptor cofactor androgen receptor trapped Clone-27. Mol Endocrinol. 2007:21(12):2864-76.

28. Chen K, Zeng J, Xiao H, Huang C, Hu J, Yao W, Yu G, Xiao W, Xu H, Ye Z. Regulation of glucose metabolism by p62/SQSTM1 through HIF1alpha. J Cell Sci. 2016;129(4):817-30.

29. Chen K, Yu G, Gumireddy K, Li A, Yao W, Gao L, Chen S, Hao J, Wang J, Huang $Q$, et al. ZBRK1, a novel tumor suppressor, activates VHL gene transcription through formation of a complex with VHL and p300 in renal cancer. Oncotarget. 2015;6(9):6959-76.

30. Chen K, Xiao H, Zeng J, Yu G, Zhou H, Huang C, Yao W, Xiao W, Hu J, Guan W, et al. Alternative splicing of EZH2 pre-mRNA by SF3B3 contributes to the tumorigenic potential of renal Cancer. Clin Cancer Res. 2017;23(13):3428-41.

31. Chen K, Huang C, Yuan J, Cheng H, Zhou R. Long-term artificial selection reveals a role of TCTP in autophagy in mammalian cells. Mol Biol Evol. 2014; 31(8):2194-211.

32. Chen $\mathrm{K}$, Chen S, Huang C, Cheng H, Zhou R. TCTP increases stability of hypoxia-inducible factor 1 alpha by interaction with and degradation of the tumour suppressor VHL. Biol Cell. 2013;105(5):208-18.

33. Liu J, Lichtenberg T, Hoadley KA, Poisson LM, Lazar AJ, Cherniack AD, Kovatich AJ, Benz CC, Levine DA, Lee AV, et al. An integrated TCGA pancancer clinical data resource to drive high-quality survival outcome analytics. Cell. 2018;173(2):400-16 e411.

34. Buttner F, Winter S, Rausch S, Reustle A, Kruck S, Junker K, Stenzl A, Agaimy A, Hartmann A, Bedke J, et al. Survival prediction of clear cell renal cell carcinoma based on gene expression similarity to the proximal tubule of the nephron. Eur Urol. 2015;68(6):1016-20.

35. Su SK, Li CY, Lei PJ, Wang X, Zhao QY, Cai Y, Wang Z, Li L, Wu M. The EZH1-SUZ12 complex positively regulates the transcription of NF-kappaB target genes through interaction with UXT. J Cell Sci. 2016;129(12): 2343-53.

36. Ho TH, Kapur P, Eckel-Passow JE, Christie A, Joseph RW, Serie DJ, Cheville JC, Thompson RH, Homayoun F, Panwar $\mathrm{V}$, et al. Multicenter validation of enhancer of zeste homolog 2 expression as an independent prognostic 
marker in localized clear cell renal cell carcinoma. J Clin Oncol. 2017;35(32): 3706-13. https://doi.org/10.1200/JCO.2017.73.3238.

37. Sakurai T, Bilim VN, Ugolkov AV, Yuuki K, Tsukigi M, Motoyama T, Tomita Y The enhancer of zeste homolog 2 (EZH2), a potential therapeutic target, is regulated by miR-101 in renal cancer cells. Biochem Biophys Res Commun. 2012;422(4):607-14.

38. Xu ZQ, Zhang L, Gao BS, Wan YG, Zhang XH, Chen B, Wang YT, Sun N, Fu YW. EZH2 promotes tumor progression by increasing VEGF expression in clear cell renal cell carcinoma. Clin Transl Oncol. 2015;17(1):41-9.

39. Li XH, Chavali PL, Babu MM. Capturing dynamic protein interactions. Science. 2018;359(6380):1105-6.

40. Tan CSH, Go KD, Bisteau X, Dai L, Yong CH, Prabhu N, Ozturk MB, Lim YT, Sreekumar L, Lengqvist J, et al. Thermal proximity coaggregation for system-wide profiling of protein complex dynamics in cells. Science. 2018; 359(6380):1170-7.

41. Li W, Wang L, Jiang C, Li H, Zhang K, Xu Y, Hao Q, Li M, Xue X, Qin X, et al. UXT is a novel regulatory factor of regulatory $T$ cells associated with Foxp3. Eur J Immunol. 2014;44(2):533-44.

42. Kim KH, Roberts CW. Targeting EZH2 in cancer. Nat Med. 2016;22(2):128-34.

43. Comet I, Riising EM, Leblanc B, Helin K. Maintaining cell identity: PRC2mediated regulation of transcription and cancer. Nat Rev Cancer. 2016; 16(12):803-10

44. Cao R, Wang L, Wang H, Xia L, Erdjument-Bromage H, Tempst P, Jones RS, Zhang Y. Role of histone H3 lysine 27 methylation in Polycomb-group silencing. Science. 2002;298(5595):1039-43.

45. Kim E, Kim M, Woo D-H, Shin Y, Shin J, Chang N, Oh Young T, Kim H, Rheey J, Nakano I, et al. Phosphorylation of EZH2 activates STAT3 signaling via STAT3 methylation and promotes Tumorigenicity of glioblastoma stem-like cells. Cancer Cell. 2013;23(6):839-52.

46. He A, Shen X, Ma Q, Cao J, von Gise A, Zhou P, Wang G, Marquez VE, Orkin SH, Pu WT. PRC2 directly methylates GATA4 and represses its transcriptional activity. Genes Dev. 2012;26(1):37-42.

47. Lee JM, Lee JS, Kim H, Kim K, Park H, Kim JY, Lee SH, Kim IS, Kim J, Lee M, et al. EZH2 generates a methyl degron that is recognized by the DCAF1/ DDB1/CUL4 E3 ubiquitin ligase complex. Mol Cell. 2012;48(4):572-86.

48. Gonzalez ME, Moore HM, Li X, Toy KA, Huang W, Sabel MS, Kidwell KM, Kleer CG. EZH2 expands breast stem cells through activation of $\mathrm{NOTCH} 1$ signaling. Proc Natl Acad Sci U S A. 2014;111(8):3098-103.

49. Xu K, Wu ZJ, Groner AC, He HH, Cai C, Lis RT, Wu X, Stack EC, Loda M, Liu T, et al. EZH2 oncogenic activity in castration-resistant prostate cancer cells is Polycomb-independent. Science. 2012;338(6113):1465-9.

50. Lee Shuet T, Li Z, Wu Z, Aau M, Guan P, Karuturi RKM, Liou Yih C, Yu Q. Context-specific regulation of NF-KB target gene expression by EZH2 in breast cancers. Mol Cell. 2011:43(5):798-810.

\section{Publisher's Note}

Springer Nature remains neutral with regard to jurisdictional claims in published maps and institutional affiliations.

Ready to submit your research? Choose BMC and benefit from:

- fast, convenient online submission

- thorough peer review by experienced researchers in your field

- rapid publication on acceptance

- support for research data, including large and complex data types

- gold Open Access which fosters wider collaboration and increased citations

- maximum visibility for your research: over $100 \mathrm{M}$ website views per year

At $\mathrm{BMC}$, research is always in progress.

Learn more biomedcentral.com/submissions 